\title{
STABILITY AND SENSITIVITY OF OPTIMIZATION PROBLEMS WITH FIRST ORDER STOCHASTIC DOMINANCE CONSTRAINTS*
}

\author{
DARINKA DENTCHEVA ${ }^{\dagger}$, RENÉ HENRION $^{\ddagger}$, AND ANDRZEJ RUSZCZYŃSKI ${ }^{\S}$
}

\begin{abstract}
We analyze the stability and sensitivity of stochastic optimization problems with stochastic dominance constraints of first order. We consider general perturbations of the underlying probability measures in the space of regular measures equipped with a suitable discrepancy distance. We show that the graph of the feasible set mapping is closed under rather general assumptions. We obtain conditions for the continuity of the optimal value and upper-semicontinuity of the optimal solutions, as well as quantitative stability estimates of Lipschitz type. Furthermore, we analyze the sensitivity of the optimal value and obtain upper and lower bounds for the directional derivatives of the optimal value. The estimates are formulated in terms of the dual utility functions associated with the dominance constraints.
\end{abstract}

Key words. stochastic programming, stochastic ordering, semi-infinite optimization, chance constraints, Lipschitz stability, metric regularity, directional differentiability

AMS subject classifications. Primary, 90C15, 90C34, 90C48; Secondary, 46N10, 60E15, 91B06

DOI. $10.1137 / 060650118$

1. Introduction. The notion of stochastic ordering (or stochastic dominance of first order) was introduced in statistics in [14, 13] and further applied and developed in economics $[17,7,6]$. It is defined as follows. For a random variable $X$ we consider its distribution function, $F(X ; \eta)=P[X \leq \eta], \eta \in \mathbb{R}$. We say that a random variable $X$ dominates in the first order a random variable $Y$ if

$$
F(X ; \eta) \leq F(Y ; \eta) \quad \forall \eta \in \mathbb{R} .
$$

We denote this relation $X \succeq_{(1)} Y$. For a modern perspective on stochastic orders, see $[15,25]$.

Let $g: \mathbb{R}^{n} \times \mathbb{R}^{s} \rightarrow \mathbb{R}$ be continuous with respect to both arguments, and let $V$ be an $s$-dimensional random vector, defined on a certain probability space $(\Omega, \mathscr{F}, P)$. For every $z \in \mathbb{R}^{n}$

$$
X_{z}(\omega)=g(z, V(\omega)), \quad \omega \in \Omega
$$

is a random variable. Given a benchmark random variable $Y$ (defined on the same probability space), an optimization model with first order stochastic dominance constraint is formulated as follows:

$$
\begin{array}{cl}
\min & f(z) \\
\text { s.t. } & X_{z} \succeq(1) \\
& z \in Z,
\end{array}
$$

*Received by the editors January 16, 2006; accepted for publication (in revised form) September 12, 2006; published electronically April 17, 2007. This work was supported by NSF awards DMS0303545, DMS-0303728, DMI-0354500, DMI-0354678, DMS-0603728, DMS-0604060, and by the DFG Research Center Matheon Mathematics for Key Technologies in Berlin.

http://www.siam.org/journals/siopt/18-1/65011.html

${ }^{\dagger}$ Department of Mathematical Sciences, Stevens Institute of Technology, Castle Point on Hudson, Hoboken, NJ 07030 (ddentche@stevens.edu).

${ }^{\ddagger}$ Weierstrass Institute, Mohrenstr. 39, 10117 Berlin, Germany (henrion@wias-berlin.de).

$\S$ Department of Management Science and Information Systems, Rutgers University, 94 Rockafeller Rd., Piscataway, NJ 08854 (rusz@business.rutgers.edu). 
where $f: \mathbb{R}^{n} \rightarrow \mathbb{R}$ and $Z \subset \mathbb{R}^{n}$. Using definition (1.1), we can express the dominance constraint as a continuum of probabilistic constraints:

$$
P[g(z, V) \geq \eta] \geq P[Y \geq \eta], \quad \eta \in \mathbb{R} .
$$

In [5] optimality conditions for a relaxation of problem (1.2) were investigated, in which the dominance constraint was enforced on an interval $[a, b]$ rather than on the entire real line:

$$
\begin{aligned}
\min & f(z) \\
\text { s.t. } & P[g(z, V) \geq \eta] \geq P[Y \geq \eta], \quad \eta \in[a, b], \\
& z \in Z .
\end{aligned}
$$

The restriction of the range of $\eta$ to a compact interval is motivated by the need to satisfy a constraint qualification condition for the problem (see Definition 2.4). Both probability functions in problem (1.3) converge to 0 when $\eta \rightarrow \infty$ and to 1 when $\eta \rightarrow \infty$, which precludes Robinson-type conditions on the whole real line.

From now on, we shall assume that $f$ is continuous and $Z$ is a nonempty closed convex set. Our objective is to investigate the stability and sensitivity of the optimal value, the feasible set, and solution set, respectively, of problem (1.3) when the random variables $V$ and $Y$ are subject to perturbations.

For the purpose of our analysis it is convenient to formulate the dominance constraint with the use of " $\geq$ " inequalities, as in (1.3). When the distributions are continuous, this formulation is equivalent to the formulation used in [5].

Problems with stochastic dominance constraints are new optimization models involving risk aversion (see $[3,4,5]$ ). As problems with a continuum of constraints on probability, they pose specific analytical and computational challenges. The probabilistic nature of the problem prevents the direct application of the theory of semiinfinite optimization. On the other hand, the specific structure of dominance constraints is significantly different from the structure of finitely many probabilistic constraints. Our stability analysis follows similar patterns to those in $[8,22,23]$, where the focus was on probabilistic constraints. However, a straightforward application of those results (a recent overview of which can be found in [21]) is not possible due to the specific structure of problem (1.3). First, in (1.3) we deal with two separate probability terms due to the consideration of a benchmark variable. Second, and more importantly, problem (1.3) has a continuum of constraints which requires a more sophisticated analysis than the case of a finite family of constraints.

In section 2, we establish the closedness of the feasible set mapping, and we obtain stability results for the optimal value, for the feasible set, and for the solution set. In section 3, we analyze the sensitivity of the optimal value function, and we obtain bounds for its directional derivatives.

2. Stability. It is obvious from the formulation of the dominance constraint that only the distribution laws of $V$ and $Y$ matter there. Therefore, we introduce the measures $\mu_{0}$ on $\mathbb{R}^{s}$ and $\nu_{0}$ on $\mathbb{R}$ induced by $V$ and $Y$. For all Borel sets $A \subset \mathbb{R}^{s}$ and $B \subset \mathbb{R}$,

$$
\begin{aligned}
& \mu_{0}(A)=P[V \in A], \\
& \nu_{0}(B)=P[Y \in B] .
\end{aligned}
$$

We denote the set of probability measures on $\mathbb{R}^{m}$ by $\mathscr{P}\left(\mathbb{R}^{m}\right)$. 
Furthermore, we introduce the multifunction $H: \mathbb{R}^{n} \times \mathbb{R} \rightrightarrows \mathbb{R}^{s}$ defined by

$$
H(z, \eta):=\left\{v \in \mathbb{R}^{s}: g(z, v) \geq \eta\right\} .
$$

We consider the following parametric optimization problem:

$$
\begin{aligned}
\min & f(z) \\
\text { s.t. } & \mu(H(z, \eta))-\nu([\eta, \infty)) \geq 0 \quad \forall \eta \in[a, b], \\
& z \in Z,
\end{aligned}
$$

with parameters $\mu \in \mathscr{P}\left(\mathbb{R}^{s}\right)$ and $\nu \in \mathscr{P}(\mathbb{R})$. The original problem (1.3) is obtained when $(\mu, \nu)=\left(\mu_{0}, \nu_{0}\right)$. Our aim is to study the stability of solutions and of the optimal value to $(2.1)$ under small perturbations of the underlying distributions $\mu_{0}$ and $\nu_{0}$.

For this purpose we equip the space $\mathscr{P}(\mathbb{R})$ with the Kolmogorov distance function:

$$
\alpha_{1}\left(\nu_{1}, \nu_{2}\right)=\sup _{\eta \in \mathbb{R}}\left|\nu_{1}([\eta, \infty))-\nu_{2}([\eta, \infty))\right| .
$$

To introduce a distance function on $\mathscr{P}\left(\mathbb{R}^{s}\right)$, which is appropriate for our problem, we define the family of sets:

$$
\mathscr{B}:=\{H(z, \eta): z \in Z, \eta \in[a, b]\} \cup\left\{v+\mathbb{R}_{-}^{s}: v \in \mathbb{R}^{s}\right\} .
$$

The distance function on $\mathscr{P}\left(\mathbb{R}^{s}\right)$ is defined as the discrepancy

$$
\alpha_{\mathrm{B}}\left(\mu_{1}, \mu_{2}\right):=\sup _{B \in \mathrm{B}}\left|\mu_{1}(B)-\mu_{2}(B)\right| .
$$

On the product space $\mathscr{P}\left(\mathbb{R}^{s}\right) \times \mathscr{P}(\mathbb{R})$ we introduce the natural distance:

$$
\alpha\left(\left(\mu_{1}, \nu_{1}\right),\left(\mu_{2}, \nu_{2}\right)\right):=\max \left\{\alpha_{\mathrm{B}}\left(\mu_{1}, \mu_{2}\right), \alpha_{1}\left(\nu_{1}, \nu_{2}\right)\right\} .
$$

Note that $\alpha$ is a metric, because the measures are compared, in particular, on all the cells of form $z+\mathbb{R}_{-}^{s}$ and $(-\infty, \eta)$, respectively.

We consider the constraint set mapping $\Phi: \mathscr{P}\left(\mathbb{R}^{s}\right) \times \mathscr{P}(\mathbb{R}) \rightrightarrows \mathbb{R}^{n}$, which assigns to every parameter $(\mu, \nu)$ the feasible set of problem $(2.1)$, i.e.,

$$
\Phi(\mu, \nu):=\{z \in Z: \mu(H(z, \eta))-\nu([\eta, \infty)) \geq 0 \quad \forall \eta \in[a, b]\} .
$$

Given any open subset $U \subseteq \mathbb{R}^{n}$, we define the $U$-localized optimal value function, $\varphi_{U}: \mathscr{P}\left(\mathbb{R}^{s}\right) \times \mathscr{P}(\mathbb{R}) \rightarrow \overline{\mathbb{R}}$, of problem (2.1) as follows:

$$
\varphi_{U}(\mu, \nu):=\inf \{f(z): z \in \Phi(\mu, \nu) \cap \operatorname{cl} U\} .
$$

The $U$-localized solution set mapping $\Psi_{U}: \mathscr{P}\left(\mathbb{R}^{s}\right) \times \mathscr{P}(\mathbb{R}) \rightrightarrows \mathbb{R}^{n}$ of problem (2.1) is defined by

$$
\Psi_{U}(\mu, \nu):=\left\{z \in \Phi(\mu, \nu) \cap \operatorname{cl} U: f(z)=\varphi_{U}(\mu, \nu)\right\} .
$$

When $U=\mathbb{R}^{n}$ we simply write $\varphi(\mu, \nu)$ and $\Psi(\mu, \nu)$.

The reason to consider localized mappings is that we allow general perturbations of the probability distributions. Then, without additional compactness conditions, no reasonable constraint qualification formulated at the solution points of the original problem (1.3) could guarantee stability of the global solution set mapping $\Psi:=\Psi_{\mathbb{R}^{n}}$.

We recall a general stability result from [10, Proposition 1 and Theorem 1] in a version adapted to our setting. In the theorem below, the symbol $\mathbb{B}(z, r)$ denotes the ball about $z$ of radius $r$. 
THEOREM 2.1. Let the following assumptions be satisfied in (2.1):

1. The original solution set $\Psi\left(\mu_{0}, \nu_{0}\right)$ is nonempty and bounded.

2. The graph of the constraint set mapping $\Phi$ is closed.

3. At every solution $z^{0} \in \Psi\left(\mu_{0}, \nu_{0}\right)$ of the original problem, there exist $\varepsilon>0$ and $L>0$ such that for all $(\mu, \nu) \in \mathbb{B}\left(\left(\mu_{0}, \nu_{0}\right) ; \varepsilon\right)$ the constraint set mapping satisfies the following two Lipschitz-like estimates:

$$
\begin{aligned}
& d\left(z, \Phi\left(\mu_{0}, \nu_{0}\right)\right) \leq L \alpha\left((\mu, \nu),\left(\mu_{0}, \nu_{0}\right)\right) \quad \forall z \in \Phi(\mu, \nu) \cap \mathbb{B}\left(z^{0} ; \varepsilon\right), \\
& d(z, \Phi(\mu, \nu)) \leq L \alpha\left((\mu, \nu),\left(\mu_{0}, \nu_{0}\right)\right) \quad \forall z \in \Phi\left(\mu_{0}, \nu_{0}\right) \cap \mathbb{B}\left(z^{0} ; \varepsilon\right) .
\end{aligned}
$$

4. $f$ is locally Lipschitz.

Then, for any bounded and open set $Q$ containing the original solution set, the following stability properties hold true:

- $\exists \delta^{\prime}>0: \Psi_{Q}(\mu, \nu) \neq \emptyset$ for all $(\mu, \nu) \in \mathbb{B}\left(\left(\mu_{0}, \nu_{0}\right) ; \delta^{\prime}\right)$.

- $\Psi_{Q}$ is upper semicontinuous at $\left(\mu_{0}, \nu_{0}\right)$ in the sense of Berge; i.e., for all open $V \supseteq \Psi\left(\mu_{0}, \nu_{0}\right)=\Psi_{Q}\left(\mu_{0}, \nu_{0}\right)$ there exists some $\delta_{V}>0$ such that

$$
\Psi_{Q}(\mu, \nu) \subseteq V \quad \forall(\mu, \nu) \in \mathbb{B}\left(\left(\mu_{0}, \nu_{0}\right) ; \delta_{V}\right) .
$$

- $\varphi_{Q}$ is continuous at $\left(\mu_{0}, \nu_{0}\right)$ and satisfies the following Lipschitz-like estimate for some constants $\delta^{*}, L^{*}>0$ :

$$
\left|\varphi_{Q}(\mu, \nu)-\varphi_{Q}\left(\mu_{0}, \nu_{0}\right)\right| \leq L^{*} \alpha\left((\mu, \nu),\left(\mu_{0}, \nu_{0}\right)\right) \quad \forall(\mu, \nu) \in \mathbb{B}\left(\left(\mu_{0}, \nu_{0}\right) ; \delta^{*}\right) .
$$

We note that the first two assertions of the theorem already follow from [19, Theorem 4.3]. In the following we want to provide verifiable conditions for the assumptions of Theorem 2.1. As far as assumption 1 is concerned, it is of a purely technical nature and may be difficult to verify in the general setting. If, however, the abstract part $Z$ of the constraint set in (2.1) happens to be compact, as is the case in many applied problems, then, of course, the boundedness assumption 1 in Theorem 2.1 is trivially satisfied. In this situation, one can even drop the localizations $\varphi_{Q}$ and $\Psi_{Q}$ in the statement of Theorem 2.1 and formulate the corresponding conclusions for the global optimal value function $\varphi$ and the global solution set mapping $\Psi$. Indeed, as one may choose $Q$ in Theorem 2.1 by compactness of $Z$ such that $Q \supseteq Z \supseteq \Psi\left(\mu_{0}, \nu_{0}\right)$, it follows that

$$
\Psi_{Q}(\mu, \nu)=\Psi(\mu, \nu)(\subset Z \subset Q) \quad \text { and } \quad \varphi_{Q}(\mu, \nu)=\varphi(\mu, \nu) \quad \forall(\mu, \nu) .
$$

Passing to assumption 2 in Theorem 2.1, this is generally satisfied under the data assumptions made for problem (1.3). To show this, we first adapt a result of [22].

Lemma 2.2. Assume that a multifunction $S: \mathbb{R}^{n} \rightrightarrows \mathbb{R}^{s}$ has a closed graph. Let $\bar{x} \in \mathbb{R}^{n}$ be such that $S(\bar{x}) \neq \emptyset$. Then for every nonnegative regular measure $\mu$ on $\mathbb{R}^{s}$ and for every $\varepsilon>0$ there exists $\delta>0$ such that

$$
\mu(S(x)) \leq \mu(S(\bar{x}))+\varepsilon, \text { whenever }\|x-\bar{x}\| \leq \delta .
$$

Proof. By the closedness of the graph,

$$
S(\bar{x})=\bigcap_{\delta>0} \operatorname{cl}\left(\bigcup_{\|x-\bar{x}\| \leq \delta} S(x)\right) .
$$


Therefore, for every regular measure $\mu$,

$$
\mu(S(\bar{x}))=\inf _{\delta>0} \mu\left(\operatorname{cl}\left(\bigcup_{\|x-\bar{x}\| \leq \delta} S(x)\right)\right) .
$$

Consequently, for every $\varepsilon>0$ there exists $\delta>0$ such that

$$
\mu(S(\bar{x}))+\varepsilon \geq \mu\left(\operatorname{cl}\left(\bigcup_{\|x-\bar{x}\| \leq \delta} S(x)\right)\right) .
$$

This implies the result.

THEOREM 2.3. The graph of the feasible set mapping $\Phi$ is closed.

Proof. Consider a sequence $\left(\mu^{n}, \nu^{n}, z^{n}\right)$ of the elements of the graph, which is convergent to some $(\bar{\mu}, \bar{\nu}, \bar{z})$ in the space $\mathscr{P}\left(\mathbb{R}^{s}\right) \times \mathscr{P}(\mathbb{R}) \times \mathbb{R}^{n}$. Since $z^{n} \in \Phi\left(\mu^{n}, \nu^{n}\right)$, then $z^{n} \in Z$ and

$$
\mu^{n}\left(H\left(z^{n}, \eta\right)\right)-\nu^{n}([\eta, \infty)) \geq 0 \quad \forall \eta \in[a, b] .
$$

As $Z$ is closed, $\bar{z} \in Z$. By the definition of $\alpha_{1}(\cdot, \cdot)$, it follows that

$$
\nu^{n}([\eta, \infty)) \rightarrow \bar{\nu}([\eta, \infty)) \quad \forall \eta \in[a, b] .
$$

Let us consider the first term in (2.6). For a fixed $\eta \in[a, b]$ we have the inequality

$$
\begin{aligned}
& \mu^{n}\left(H\left(z^{n}, \eta\right)\right)-\bar{\mu}(H(\bar{z}, \eta)) \\
= & {\left[\mu^{n}\left(H\left(z^{n}, \eta\right)\right)-\bar{\mu}\left(H\left(z^{n}, \eta\right)\right)\right]+\left[\bar{\mu}\left(H\left(z^{n}, \eta\right)\right)-\bar{\mu}(H(\bar{z}, \eta))\right] } \\
\leq & \alpha_{\mathrm{B}}\left(\mu^{n}, \bar{\mu}\right)+\left[\bar{\mu}\left(H\left(z^{n}, \eta\right)\right)-\bar{\mu}(H(\bar{z}, \eta))\right] .
\end{aligned}
$$

By assumption, $\alpha_{\mathrm{B}}\left(\mu^{n}, \bar{\mu}\right) \rightarrow 0$, and we can focus on the term in brackets. By the continuity of $g$, the multifunction $H(\cdot, \eta)$ has a closed graph. We now apply Lemma 2.2 to conclude that for every $\varepsilon>0$ there exists $\delta>0$ such that

$$
\bar{\mu}(H(z, \eta)) \leq \bar{\mu}(H(\bar{z}, \eta))+\varepsilon, \text { whenever }\|z-\bar{z}\| \leq \delta .
$$

For all sufficiently large $n$ one has $\left\|z^{n}-\bar{z}\right\| \leq \delta$ and therefore

$$
\bar{\mu}\left(H\left(z^{n}, \eta\right)\right) \leq \bar{\mu}(H(\bar{z}, \eta))+\varepsilon .
$$

Passing to the limit with $n \rightarrow \infty$ and noting that $\varepsilon>0$ was arbitrary, we obtain

$$
\limsup _{n \rightarrow \infty} \bar{\mu}\left(H\left(z^{n}, \eta\right)\right) \leq \bar{\mu}(H(\bar{z}, \eta)) .
$$

Combining relations (2.8) and (2.9), we conclude that

$$
\limsup _{n \rightarrow \infty} \mu^{n}\left(H\left(z^{n}, \eta\right)\right) \leq \bar{\mu}(H(\bar{z}, \eta)) .
$$

Using this in (2.6), with a view to (2.7), we obtain

$$
\begin{aligned}
\bar{\mu}(H(\bar{z}, \eta))-\bar{\nu}(\eta, \infty)) & \geq \limsup _{n \rightarrow \infty} \mu^{n}\left(H\left(z^{n}, \eta\right)\right)-\lim _{n \rightarrow \infty} \nu^{n}([\eta, \infty)) \\
& =\limsup _{n \rightarrow \infty}\left[\mu^{n}\left(H\left(z^{n}, \eta\right)\right)-\nu^{n}([\eta, \infty))\right] \geq 0 .
\end{aligned}
$$


Since $\eta$ was arbitrary, we obtain the relation

$$
\bar{\mu}(H(\bar{z}, \eta))-\bar{\nu}(\eta, \infty)) \geq 0 \quad \forall \eta \in[a, b] .
$$

This amounts to $\bar{z} \in \Phi(\bar{\mu}, \bar{\nu})$, as desired.

Remark 1. Let us observe that we did not use the compactness of the set $[a, b]$ in the proof, and therefore Theorem 2.3 holds true for the dominance relation enforced on the whole real line.

The verification of assumption 3 in Theorem 2.1 is less direct and will be based on an appropriate constraint qualification for problem (2.1) at the original parameter $\left(\mu_{0}, \nu_{0}\right)$. To formulate this constraint qualification, we assume the following differential uniform dominance condition introduced in [5].

Definition 2.4. Problem (2.1) for $\mu=\mu_{0}$ and $\nu=\nu_{0}$ satisfies the differential uniform dominance condition at the point $z^{0} \in Z$ if

(i) $\mu_{0}(H(z, \eta))$ is continuous with respect to $\eta$ in $[a, b]$, differentiable with respect to $z$ in a neighborhood of $z^{0}$ for all $\eta \in[a, b]$, and its derivative is jointly continuous with respect to both arguments;

(ii) $\nu_{0}([\cdot, \infty))$ is continuous;

(iii) there exists $z^{1} \in Z$ such that

$$
\min _{a \leq \eta \leq b}\left\{\mu_{0}\left(H\left(z^{0}, \eta\right)\right)+\nabla_{z} \mu_{0}\left(H\left(z^{0}, \eta\right)\right)\left(z^{1}-z^{0}\right)-\nu_{0}([\eta, \infty))\right\}>0 .
$$

The differentiability assumptions on $\mu_{0}(H(\cdot, \eta))$ can be guaranteed by assuming continuous differentiability of the function $g$ with respect to both arguments, the existence of the probability density of the random vector $V$, and by mild regularity conditions (see [9]). Then

$$
\nabla_{z} \mu_{0}(H(z, \eta))=\int_{\partial H(z, \eta)} \frac{\varphi(v)}{\left\|\nabla_{v} g(z, v)\right\|} \nabla_{z} g(z, v) \lambda(d v),
$$

where $\partial H(z, \eta)$ is the surface of the set $H(z, \eta)$ and $\lambda$ is the surface Lebesgue measure. The regularity conditions mentioned require that the gradient $\nabla_{v} g(z, v)$ be nonzero and that the integrand above be uniformly bounded (in a neighborhood of $z$ ) by an integrable function.

For example, if $g(z, V)=\langle z, V\rangle$ and $V$ has a nondegenerate multivariate normal distribution $\mathscr{N}(\bar{v}, \Sigma)$, then

$$
\mu_{0}(H(z, \eta))=1-\Phi\left(\frac{\eta-\langle z, \bar{v}\rangle}{\sqrt{\langle z, \Sigma z\rangle}}\right)
$$

where $\Phi(\cdot)$ is the distribution function of the standard normal variable. In this case condition (i) of Definition 2.4 is satisfied at every $z \neq 0$.

The differential uniform dominance condition has substantial consequences. Let $\mathscr{C}$ be the Banach space of continuous functions on $[a, b]$. Consider the mapping $\Gamma: \mathbb{R}^{n} \rightarrow \mathscr{C}$ defined as

$$
\Gamma(z)(\eta)=\mu_{0}(H(z, \eta))-\nu_{0}([\eta, \infty)), \quad \eta \in[a, b],
$$

where $\nu_{0}([\cdot, \infty)) \in \mathscr{C}$. Denote by $K$ the nonnegative cone in $\mathscr{C}$.

LEMMA 2.5. Assume that $\mu_{0}(H(z, \eta))$ is continuously differentiable with respect to $z$ in a neighborhood of $z^{0} \in Z$ and for all $\eta \in[a, b], \mu_{0}(H(z, \cdot))$ is continuous in 
$[a, b]$, and $\Gamma\left(z^{0}\right) \in K$. The differential uniform dominance condition is satisfied at $z^{0}$ if and only if the multifunction

$$
z \mapsto \begin{cases}\Gamma(z)-K & \text { if } z \in Z, \\ \emptyset & \text { otherwise }\end{cases}
$$

is metrically regular at $\left(z^{0}, 0\right)$.

Proof. We observe that the differential uniform dominance condition is equivalent to Robinson's constraint qualification condition (see [18])

$$
0 \in \operatorname{int}\left\{\Gamma\left(z^{0}\right)+\nabla_{z} \Gamma\left(z^{0}\right)\left(Z-z^{0}\right)-K\right\} .
$$

Indeed, it is easy to see that the uniform dominance condition implies Robinson's condition. On the other hand, if Robinson's condition holds true, then there exists $\varepsilon>0$ such that the function identically equal to $\varepsilon$ is an element of the set on the right-hand side of (2.11). Then we can find $z^{1}$ such that

$$
\Gamma\left(z^{0}\right)(\eta)+\left[\nabla_{z} \Gamma\left(z^{0}\right)(\eta)\right]\left(z^{1}-z^{0}\right) \geq \varepsilon \quad \forall \eta \in[a, b] .
$$

Consequently, the uniform dominance condition is satisfied. On the other hand, Robinson's constraint qualification at $z^{0}$ is equivalent to the metric regularity of $(2.10)$ at $\left(z^{0}, 0\right)$ (see $\left.[2]\right)$.

The next proposition shows that the verification of assumption 3 in Theorem 2.1 can be reduced to the differential uniform dominance condition.

Proposition 2.6. Let the differential uniform dominance condition be satisfied at some $z^{0} \in \Phi\left(\mu_{0}, \nu_{0}\right)$. Then relations (2.3) and (2.4) of Theorem 2.1 hold true at $z^{0}$.

Proof. We introduce the multifunction $M: \mathscr{C} \rightrightarrows \mathbb{R}^{n}$ as the following parameter dependent constraint set mapping:

$$
M(w):=\left\{z \in Z: \mu_{0}(H(z, \eta))-w(\eta) \geq 0 \forall \eta \in[a, b]\right\} .
$$

(The relation between $M$ and $\Phi$ is given by $\Phi\left(\mu_{0}, \nu\right)=M(\nu([\cdot, \infty)))$ for all continuous distributions $\nu \in \mathscr{P}(\mathbb{R})$.) Define $w_{0}(\cdot)=\nu_{0}([\cdot, \infty))$. By assumption, $w_{0} \in \mathscr{C}$.

By Lemma 2.5, the differential uniform dominance condition is equivalent to metric regularity of $(2.10)$ at $\left(z^{0}, 0\right)$, which, upon passing to the inverse multifunction, is equivalent to the pseudo-Lipschitz property of $M$ at $\left(w^{0}, z^{0}\right)$ (see, e.g., [12, Lemma 1.12] and [20, Theorem 9.43]). Accordingly, there exist $\tilde{\varepsilon}>0$ and $\tilde{L}>0$ such that

$$
d\left(z, M\left(w_{2}\right)\right) \leq \tilde{L} d\left(w_{1}, w_{2}\right) \quad \forall z \in M\left(w_{1}\right) \cap \mathbb{B}\left(z^{0} ; \tilde{\varepsilon}\right) \forall w_{1}, w_{2} \in \mathbb{B}\left(w_{0} ; \tilde{\varepsilon}\right),
$$

where the last ball is taken in the metric of $\mathscr{C}$. First, we verify the following chain of inclusions for all $(\mu, \nu) \in \mathscr{P}\left(\mathbb{R}^{s}\right) \times \mathscr{P}(\mathbb{R})$ :

$$
M\left(w_{0}+2 \alpha\left((\mu, \nu),\left(\mu_{0}, \nu_{0}\right)\right) \cdot \mathbb{1}\right) \subseteq \Phi(\mu, \nu) \subseteq M\left(w_{0}-2 \alpha\left((\mu, \nu),\left(\mu_{0}, \nu_{0}\right)\right) \cdot \mathbb{1}\right)
$$

where $\mathbb{1}$ is the function on $[a, b]$ taking the constant value 1 . Note that $M$ is applied to continuous functions as required. Now, if

$$
z \in M\left(w_{0}+2 \alpha_{\mathrm{B}}\left((\mu, \nu),\left(\mu_{0}, \nu_{0}\right)\right) \cdot \mathbb{1}\right),
$$


then $z \in Z$ and, by definition of $\alpha$,

$$
\begin{aligned}
0 & \leq \mu_{0}(H(z, \eta))-\left(w_{0}(\eta)+2 \alpha\left((\mu, \nu),\left(\mu_{0}, \nu_{0}\right)\right)\right) \\
& \left.=\mu_{0}(H(z, \eta))-\nu_{0}([\eta, \infty))-2 \alpha\left((\mu, \nu),\left(\mu_{0}, \nu_{0}\right)\right)\right) \\
& \leq \mu(H(z, \eta))-\nu([\eta, \infty)) \quad \forall \eta \in[a, b] .
\end{aligned}
$$

This establishes the first inclusion of (2.13), and the second one is completely analogous.

In order to check $(2.3)$, let $(\mu, \nu) \in \mathbb{B}\left(\left(\mu_{0}, \nu_{0}\right) ; \tilde{\varepsilon} / 2\right)$ and $z \in \Phi(\mu, \nu) \cap \mathbb{B}\left(z^{0} ; \tilde{\varepsilon} / 2\right)$ be arbitrary. Define $w_{1} \in \mathscr{C}$ by $w_{1}:=w_{0}-2 \alpha\left((\mu, \nu),\left(\mu_{0}, \nu_{0}\right)\right) \cdot \mathbb{1}$. Then the second inclusion of (2.13) entails that $z \in M\left(w_{1}\right)$. Furthermore,

$$
d\left(w_{1}, w_{0}\right)=2 \alpha\left((\mu, \nu),\left(\mu_{0}, \nu_{0}\right)\right) \leq \tilde{\varepsilon}
$$

Consequently, we may apply (2.12) to $w_{1}$ and to $w_{2}:=w_{0} \in \mathscr{C}$ :

$$
d\left(z, \Phi\left(\mu_{0}, \nu_{0}\right)\right)=d\left(z, M\left(w_{0}\right)\right) \leq \tilde{L} d\left(w_{1}, w_{0}\right)=2 \tilde{L} \alpha\left((\mu, \nu),\left(\mu_{0}, \nu_{0}\right)\right) .
$$

Therefore, (2.3) holds true with $L:=2 \tilde{L}$ and $\varepsilon:=\tilde{\varepsilon} / 2$. As for (2.4), take arbitrary $(\mu, \nu) \in \mathbb{B}\left(\left(\mu_{0}, \nu_{0}\right) ; \tilde{\varepsilon} / 2\right)$ and $z \in \Phi\left(\mu_{0}, \nu_{0}\right) \cap \mathbb{B}\left(z^{0} ; \tilde{\varepsilon} / 2\right)$. Define $w_{2} \in \mathscr{C}$ by $w_{2}:=$ $w_{0}+2 \alpha\left((\mu, \nu),\left(\mu_{0}, \nu_{0}\right)\right) \cdot \mathbb{1}$. Then

$$
d\left(w_{2}, w_{0}\right)=2 \alpha\left((\mu, \nu),\left(\mu_{0}, \nu_{0}\right)\right) \leq \tilde{\varepsilon},
$$

and we may apply (2.12) to $w_{1}:=w_{0}$ and to $w_{2}$. Further taking into account the first inclusion of (2.13), one arrives at

$$
d(z, \Phi(\mu, \nu)) \leq d\left(z, M\left(w_{2}\right) \leq \tilde{L} d\left(w_{0}, w_{2}\right)=2 \tilde{L} \alpha\left((\mu, \nu),\left(\mu_{0}, \nu_{0}\right)\right),\right.
$$

which is (2.4) with the same values $L:=2 \tilde{L}$ and $\varepsilon:=\tilde{\varepsilon} / 2$ as for (2.3).

\section{Sensitivity of the optimal value.}

3.1. Optimality conditions. In order to analyze the sensitivity of the optimal value function, we need to briefly recall optimality conditions for problem (1.3). From now on we assume that $f$ is continuously differentiable.

We define the set $\mathscr{U}([a, b])$ of functions $u(\cdot)$ satisfying the following conditions:

$$
\begin{aligned}
& u(\cdot) \text { is nondecreasing and right continuous; } \\
& u(t)=0 \forall t \leq a ; \\
& u(t)=u(b) \forall t \geq b .
\end{aligned}
$$

It is evident that $\mathscr{U}([a, b])$ is a convex cone. The slight difference from the definition of the set $\mathscr{U}$ introduced in [5] is due to the fact that we formulate the stochastic dominance constraint in (1.3) via the $\geq$ inequality.

We introduce the functional $L: \mathbb{R}^{n} \times \mathscr{U}([a, b]) \times \mathscr{P}\left(\mathbb{R}^{s}\right) \times \mathscr{P}(\mathbb{R}) \rightarrow \mathbb{R}$ associated with problem (1.3):

$$
L(z, u ; \mu, \nu):=f(z)-\int u(g(z, v)) \mu(d v)+\int u(y) \nu(d y) .
$$

As shown in [5], the functional $L$ plays a similar role to that of a Lagrangian of the problem. 
THEOREM 3.1. Assume that the differential uniform dominance condition is satisfied at a local minimum $\hat{z}$ of problem (1.3). Then there exists a function $\hat{u} \in$ $\mathscr{U}([a, b])$ such that

$$
\begin{gathered}
-\nabla_{z} L\left(\hat{z}, \hat{u} ; \mu_{0}, \nu_{0}\right) \in N_{Z}(\hat{z}), \\
\int \hat{u}(g(\hat{z}, v)) \mu_{0}(d v)=\int \hat{u}(y) \nu_{0}(d y) .
\end{gathered}
$$

The proof follows the same line of argument as the proof in [5] and is omitted here. It uses the correspondence between a nonnegative measure $\lambda$ on $[a, b]$ and a function $u \in \mathscr{U}([a, b])$ :

$$
u(\eta)=\lambda([a, \eta]), \quad \eta \in[a, b] .
$$

Remark 2. The set $\hat{U}(\hat{z})$ of functions in $\mathscr{U}([a, b])$ satisfying (3.2)-(3.3) for the local minimum $\hat{z}$ is convex, bounded, and weakly* closed in the following sense: if a sequence of functions $u^{k} \in \hat{U}(\hat{z})$ and $u \in \mathscr{U}([a, b])$ are such that

$$
\lim _{k \rightarrow \infty} \int_{a}^{b} c(\eta) d u^{k}(\eta)=\int_{a}^{b} c(\eta) d u(\eta) \quad \forall c \in \mathscr{C},
$$

then $u \in \hat{U}(\hat{z})$. This follows from [1, Theorem 3.6] and the application of (3.4).

If the function $g(\cdot, \cdot)$ is quasi-concave and $\mu$ has an $r$-concave probability density function, with $r \geq-1 / s$, then the feasible set of problem (1.3) is convex (see [16]). Therefore we can formulate the following sufficient conditions of optimality, as in [5].

TheOREm 3.2. Assume that a point $\hat{z}$ is feasible for problem (1.3). Suppose that there exists a function $\hat{u} \in \mathscr{U}([a, b])$ such that conditions (3.2)-(3.3) are satisfied. If the function $f$ is convex, the function $g(\cdot, \cdot)$ is quasi-concave, and $V$ has an $r$-concave probability density function, with $r \geq-1 / s$, then $\hat{z}$ is an optimal solution of problem (1.3).

Let us observe that under the assumptions of Theorem 3.2 the functional (3.1) is, in general, not a quasi-convex function of $z$.

3.2. Upper bound. Consider the measures

$$
\begin{aligned}
\mu_{t} & =\mu_{0}+t \gamma, \\
\nu_{t} & =\nu_{0}+t \sigma,
\end{aligned}
$$

where $\gamma$ and $\sigma$ are regular signed measures on $\mathbb{R}^{s}$ and $\mathbb{R}$, respectively, and $t>0$. We shall bound the optimal value $\varphi\left(\mu_{t}, \nu_{t}\right)$ of the perturbed problem

$$
\begin{aligned}
\min & f(z) \\
\text { s.t. } & \mu_{t}(H(z, \eta))-\nu_{t}([\eta, \infty)) \geq 0 \quad \forall \eta \in[a, b], \\
& z \in Z
\end{aligned}
$$

Our objective is to develop bounds for the limit of the quotients $\left[\varphi\left(\mu_{t}, \nu_{t}\right)-\varphi\left(\mu_{0}, \nu_{0}\right)\right] / t$, when $t \downarrow 0$.

TheOREM 3.3. Let $\hat{Z}$ be the set of optimal solutions of problem (1.3). Assume the following conditions:

(i) The differential uniform dominance condition is satisfied at each point $\hat{z} \in \hat{Z}$. 
(ii) $\gamma(H(z, \eta))$ is continuous with respect to both arguments at $(\hat{z}, \eta)$ for all $\eta \in$ $[a, b]$, is differentiable with respect to $z$ in a neighborhood of each $\hat{z} \in \hat{Z}$ for every value of $\eta \in[a, b]$, and its derivative is jointly continuous with respect to both arguments.

(iii) $\sigma([\eta, \infty))$ is a continuous function of $\eta$.

Then

$$
\begin{aligned}
\limsup _{t \downarrow 0} & \frac{1}{t}\left[\varphi\left(\mu_{t}, \nu_{t}\right)-\varphi\left(\mu_{0}, \nu_{0}\right)\right] \\
& \leq \inf _{\hat{z} \in \hat{Z}} \sup _{\hat{u} \in \hat{U}(\hat{z})}\left\{\int \hat{u}(g(\hat{z}, v)) \gamma(d v)+\int \hat{u}(y) \sigma(d y)\right\},
\end{aligned}
$$

where $\hat{U}(\hat{z})$ is the set of functions in $\mathscr{U}([a, b])$ satisfying $(3.2)-(3.3)$ at the minimum $\hat{z}$.

Proof. Our result is close in spirit to that of [1, Proposition 4.22], but we work with weaker assumptions by exploiting the structure of the problem.

Fix $\hat{z} \in \hat{Z}$. We shall construct feasible points of the perturbed problem of the form

$$
\tilde{z}_{t}=\hat{z}+t h+o(t)
$$

Define the set

$$
\mathscr{A}=\left\{\eta \in[a, b]: \mu_{0}(H(\hat{z}, \eta))=\nu_{0}([\eta, \infty))\right\},
$$

and let $T_{Z}(\hat{z})$ denote the tangent cone to $Z$ at $\hat{z}$.

We assume that the direction $h$ in (3.7) is an element of the tangent cone $T_{Z}(\hat{z})$ and satisfies the infinite system of linear inequalities:

$$
\left\langle\nabla_{z} \mu_{0}(H(\hat{z}, \eta)), h\right\rangle+\gamma(H(\hat{z}, \eta))-\sigma([\eta, \infty)) \geq 0 \quad \forall \eta \in \mathscr{A} .
$$

It follows from the uniform dominance condition that there exists $\varepsilon>0$ such that

$$
\left\langle\nabla_{z} \mu_{0}(H(\hat{z}, \eta)), z^{1}-\hat{z}\right\rangle>\varepsilon
$$

for all $\eta \in \mathscr{A}$. Therefore inequalities (3.8) can be satisfied by choosing $h=\tau\left(z^{1}-\hat{z}\right)$ with a sufficiently large $\tau$.

Let $z_{t}=\hat{z}+t h$. The uniform dominance condition implies that

$$
\begin{aligned}
\mu_{t}\left(H\left(z_{t}, \eta\right)\right) & =\mu_{0}\left(H\left(z_{t}, \eta\right)\right)+t \gamma\left(H\left(z_{t}, \eta\right)\right) \\
& =\mu_{0}(H(\hat{z}, \eta))+t\left\langle\nabla_{z} \mu_{0}(H(\hat{z}, \eta)), h\right\rangle+t \gamma\left(H\left(z_{t}, \eta\right)\right)+o(t, \eta),
\end{aligned}
$$

where $o(t, \eta) / t \rightarrow 0$ as $t \rightarrow 0$, uniformly over $\eta \in[a, b]$.

We shall estimate the term $\gamma\left(H\left(z_{t}, \eta\right)\right)$ from below. Choose any $\hat{\eta} \in[a, b]$. By the continuity of $\gamma(H(z, \eta))$ around the point $(\hat{z}, \hat{\eta})$, for every $\varepsilon>0$ there exists $\delta(\varepsilon, \hat{\eta})>0$ such that

$$
\gamma(H(z, \eta)) \geq \gamma(H(\hat{z}, \hat{\eta}))-\varepsilon
$$

for all $(z, \eta)$ such that $\|z-\hat{z}\| \leq \delta(\varepsilon, \hat{\eta})$ and $|\eta-\hat{\eta}| \leq \delta(\varepsilon, \hat{\eta})$. For each $\varepsilon$ the intervals $|\eta-\hat{\eta}| \leq \delta(\varepsilon, \hat{\eta})$, where $\hat{\eta}$ runs through $[a, b]$, cover $[a, b]$. Choosing a finite subcovering, we conclude that there exists $\delta(\varepsilon)>0$ such that (3.10) holds true for all $z$ satisfying $\|z-\hat{z}\| \leq \delta(\varepsilon)$ and for all $\eta \in[a, b]$. 
Define $r(t)=\inf \{\varepsilon>0: \delta(\varepsilon) \geq t\|h\|\}$. Observe that $r(t) \rightarrow 0$ as $t \downarrow 0$. It follows from (3.10) that

$$
\gamma\left(H\left(z_{t}, \eta\right)\right) \geq \gamma(H(\hat{z}, \eta))-r(t) .
$$

Substituting this estimate into (3.9), we obtain

$$
\mu_{t}\left(H\left(z_{t}, \eta\right)\right) \geq \mu_{0}(H(\hat{z}, \eta))+t\left\langle\nabla_{z} \mu_{0}(H(\hat{z}, \eta)), h\right\rangle+t \gamma(H(\hat{z}, \eta))+o(t, \eta)-\operatorname{tr}(t) .
$$

Using condition (3.8) and the feasibility of $\hat{z}$, we conclude that

$$
\begin{aligned}
\mu_{t}\left(H\left(z_{t}, \eta\right)\right)-\nu_{t}([\eta, \infty))= & {\left[\mu_{0}(H(\hat{z}, \eta))-\nu_{0}([\eta, \infty))\right] } \\
& +t\left[\left\langle\nabla_{z} \mu_{0}(H(\hat{z}, \eta)), h\right\rangle+\gamma(H(\hat{z}, \eta))-\sigma([\eta, \infty))\right] \\
& +o(t, \eta)-\operatorname{tr}(t) \\
\geq & o(t, \eta)-\operatorname{tr}(t) \quad \forall \eta \in[a, b] .
\end{aligned}
$$

Consequently, the point $z_{t}$ may violate the constraints of the perturbed problem only by quantities which are infinitely smaller than $t$. Define the mapping $\Gamma: \mathbb{R}^{n} \times \mathbb{R} \rightarrow \mathscr{C}$ as follows:

$$
\Gamma(z, t)(\eta)=\mu_{t}(H(z, \eta))-\nu_{t}([\eta, \infty)), \quad \eta \in[a, b] .
$$

The system

$$
\begin{gathered}
\Gamma(z, t) \in K, \\
z \in Z,
\end{gathered}
$$

is stable about $(\hat{z}, 0)$ (see, e.g., [1, Theorem 2.87]). Therefore, for all sufficiently small $t>0$, we can slightly modify $z_{t}$ to get a point $\tilde{z}_{t}$ such that

$$
\begin{gathered}
\Gamma\left(\tilde{z}_{t}, t\right) \in K \\
\tilde{z}_{t} \in Z, \\
\left\|\tilde{z}_{t}-z_{t}\right\| \leq C\left[\operatorname{dist}\left(\Gamma\left(z_{t}, t\right), K\right)+\operatorname{dist}\left(z_{t}, Z\right)\right],
\end{gathered}
$$

where $C$ is some constant. Using (3.11) and the fact that $h$ is tangent to $Z$, we obtain that

$$
\lim _{t \downarrow 0} \frac{1}{t}\left(\tilde{z}_{t}-\hat{z}\right)=h
$$

As $\tilde{z}_{t}$ is feasible,

$$
\varphi\left(\mu_{t}, \nu_{t}\right) \leq f\left(\tilde{z}_{t}\right) .
$$

Subtracting $\varphi\left(\mu_{0}, \nu_{0}\right)$, dividing by $t$, and passing to the limit, we obtain

$$
\limsup _{t \downarrow 0} \frac{1}{t}\left[\varphi\left(\mu_{t}, \nu_{t}\right)-\varphi\left(\mu_{0}, \nu_{0}\right)\right] \leq \limsup _{t \downarrow 0} \frac{1}{t}\left[f\left(\tilde{z}_{t}\right)-f(\hat{z})\right]=\langle\nabla f(\hat{z}), h\rangle .
$$

It follows that the limit on the left-hand side of (3.12) is bounded from above by the optimal value of the problem

$$
\begin{aligned}
\min & \langle\nabla f(\hat{z}), h\rangle \\
\text { s.t. } & \left\langle\nabla_{z} \mu_{0}(H(\hat{z}, \eta)), h\right\rangle \geq-\gamma(H(\hat{z}, \eta))+\sigma([\eta, \infty)) \quad \forall \eta \in \mathscr{A}, \\
& h \in T_{Z}(\hat{z}) .
\end{aligned}
$$


The optimal value of the linear-conic problem (3.13) is equal to the optimal value of the following dual problem (see, e.g., [1, Theorem 5.106]):

$$
\begin{aligned}
\max _{\lambda} & \int_{a}^{b}[-\gamma(H(\hat{z}, \eta))+\sigma([\eta, \infty))] \lambda(d \eta) \\
\text { s.t. } & -\nabla f(\hat{z})-\int_{a}^{b} \nabla_{z} \mu_{0}(H(\hat{z}, \eta)) \lambda(d \eta) \in N_{Z}(\hat{z}), \\
& \quad \lambda \geq 0 .
\end{aligned}
$$

Here $\lambda$ is a regular measure on $\mathscr{A}$. Moreover, it is sufficient to consider atomic measures $\lambda$ with at most $n+1$ atoms.

Extending $\lambda$ to $[a, b]$, associating with it a function $u(\cdot)=\lambda([a, \cdot])$, and changing the order of integration, we obtain the identity

$$
\begin{aligned}
\int_{a}^{b} \gamma(H(\hat{z}, \eta)) \lambda(d \eta) & =\int_{a}^{b} \int_{v \in H(\hat{z}, \eta)} \gamma(d v) \lambda(d \eta)=\int_{a}^{b} \int_{\{v: g(\hat{z}, v) \geq \eta\}} \gamma(d v) \lambda(d \eta) \\
& =\iint_{a}^{g(\hat{z}, v)} \lambda(d \eta) \gamma(d v)=\int u(g(\hat{z}, v)) \gamma(d v) .
\end{aligned}
$$

In a similar way we transform other integrals in (3.14) to obtain the following form of the dual problem:

$$
\begin{aligned}
& \max _{u(\cdot)}-\int u(g(\hat{z}, v)) \gamma(d v)+\int u(y) \sigma(d y) \\
& \text { s.t. }-\nabla f(\hat{z})-\nabla_{z} \int u(g(\hat{z}, v)) \mu_{0}(d v) \in N_{Z}(\hat{z}), \\
& u(\cdot) \in \mathscr{U}([a, b]) \\
& u(\cdot) \text { satisfies }(3.3) .
\end{aligned}
$$

We observe that the feasible set of this problem is the set $\hat{U}$ given by (3.2)-(3.3). Now we continue the estimate (3.12) as follows:

$$
\limsup _{t \downarrow 0} \frac{1}{t}\left[\varphi\left(\mu_{t}, \nu_{t}\right)-\varphi\left(\mu_{0}, \nu_{0}\right)\right] \leq \sup _{\hat{u} \in \hat{U}(\hat{z})}\left\{-\int \hat{u}(g(\hat{z}, v)) \gamma(d v)+\int \hat{u}(y) \sigma(d y)\right\} .
$$

As $\hat{z} \in \hat{Z}$ was arbitrary, we conclude that

$$
\limsup _{t \downarrow 0} \frac{1}{t}\left[\varphi\left(\mu_{t}, \nu_{t}\right)-\varphi\left(\mu_{0}, \nu_{0}\right)\right] \leq \inf _{\hat{z} \in \hat{Z}} \sup _{\hat{u} \in \hat{U}(\hat{z})}\left\{-\int \hat{u}(g(\hat{z}, v)) \gamma(d v)+\int \hat{u}(y) \sigma(d y)\right\},
$$

which was what we set out to prove.

As discussed in the proof, it is sufficient to consider the supremum over piecewise constant functions $\hat{u} \in \hat{U}$ having at most $n+1$ jumps.

COROLlary 3.4. Suppose that $\mu_{1}=\mu_{0}+\gamma$ is a nonnegative measure and let $\nu_{1}=\nu_{0}+\sigma$. Then

$$
\limsup _{t \downarrow 0} \frac{1}{t}\left[\varphi\left(\mu_{t}, \nu_{t}\right)-\varphi\left(\mu_{0}, \nu_{0}\right)\right] \leq \inf _{\hat{z} \in \hat{Z}} \sup _{\hat{u} \in \hat{U}(\hat{z})} \int \hat{u}(y) \nu_{1}(d y) .
$$


Proof. We can rewrite the estimate (3.6) as follows:

$$
\begin{aligned}
\limsup _{t \downarrow 0} \frac{1}{t}\left[\varphi\left(\mu_{t}, \nu_{t}\right)-\varphi\left(\mu_{0}, \nu_{0}\right)\right] \leq \inf _{\hat{z} \in \hat{Z}} \sup _{\hat{u} \in \hat{U}(\hat{z})}\left\{\int \hat{u}(g(\hat{z}, v)) \mu_{0}(d v)\right. \\
\left.-\int \hat{u}(g(\hat{z}, v)) \mu_{1}(d v)+\int \hat{u}(y) \nu_{1}(d y)-\int \hat{u}(y) \nu_{0}(d y)\right\} .
\end{aligned}
$$

As the function $\hat{u}(\cdot)$ is nonnegative, we can skip the second term on the right-hand side. Using the complementarity condition (3.3), we get the required inequality.

3.3. Lower bound. Let us start from the following observation.

Lemma 3.5. Consider any measures $\mu \in \mathscr{P}\left(\mathbb{R}^{s}\right)$ and $\nu \in \mathscr{P}(\mathbb{R})$ and a point $z \in Z$ such that

$$
\mu(H(z, \eta)) \geq \nu([\eta, \infty)), \quad \eta \in[a, b] .
$$

Then for every $u \in \mathscr{U}([a, b])$ we have

$$
\int u(g(z, v)) \mu(d v) \geq \int u(y) \nu(d y) .
$$

Proof. For a function $u \in \mathscr{U}([a, b])$ we define a nonnegative measure $\lambda$ on $[a, b]$ by the relation $u(\cdot)=\lambda([a, \cdot])$. Integrating the inequalities (3.17), changing the order of integration as in (3.15), we obtain the postulated inequality.

Suppose that $u \in \mathscr{U}([a, b])$. Employing Lemma 3.5, we obtain

$$
\varphi(\mu, \nu) \geq \inf _{z \in Z}\left\{f(z)-\int u(g(z, v)) \mu(d v)\right\}+\int u(y) \nu(d y) .
$$

We get the general dual lower bound

$$
\varphi(\mu, \nu) \geq \sup _{u \in \mathrm{U}([a, b])} \inf _{z \in Z}\left\{f(z)-\int u(g(z, v)) \mu(d v)+\int u(y) \nu(d y)\right\} .
$$

In order to obtain tighter bounds we consider the perturbations in directions

$$
\begin{aligned}
\mu_{t} & =\mu_{0}+t \gamma, \\
\nu_{t} & =\nu_{0}+t \sigma .
\end{aligned}
$$

We shall develop lower bounds for the differential quotients $\left[\varphi\left(\mu_{t}, \nu_{t}\right)-\varphi\left(\mu_{0}, \nu_{0}\right)\right] / t$ when $t \downarrow 0$. Our result is similar to the standard approach employed in $[1$, Theorem 4.24]. However, it is unrealistic to assume that the Lagrangian is convex (even under the assumptions of Theorem 3.2), and that is why we need Lipschitz stability of optimal solutions.

THEOREM 3.6. Assume that $\hat{z}$ is the unique optimal solution of problem (1.3) and that the differential uniform dominance condition is satisfied at $\hat{z}$. Furthermore, assume that the perturbed problems (3.5) have solutions $z_{t}$ such that $\left\|z_{t}-\hat{z}\right\| \leq L t$ with some constant $L$. Let $\hat{U}$ be the set of functions $\hat{u}(\cdot)$ satisfying the optimality conditions (3.2)-(3.3). Then

$$
\begin{aligned}
& \liminf _{t \rightarrow 0} \frac{1}{t}\left[\varphi\left(\mu_{t}, \nu_{t}\right)-\varphi\left(\mu_{0}, \nu_{0}\right)\right] \\
& \quad \geq \sup _{\hat{u} \in \hat{U}}\left\{-\int \hat{u}(g(\hat{z}, v)) \gamma(d v)+\int \hat{u}(y) \sigma(d y)\right\} .
\end{aligned}
$$


Proof. Consider problem (2.1) and its Lagrangian

$$
\Lambda(z, \lambda ; \mu, \nu)=f(z)-\int_{a}^{b}[\mu(H(z, \eta))-\nu([\eta, \infty))] \lambda(d \eta)
$$

where $\lambda$ is a nonnegative regular measure on $[a, b]$. Fix $\mu=\mu_{0}$ and $\nu=\nu_{0}$. As in $[5]$, owing to the differential uniform dominance condition at $\hat{z}$, there exists a measure $\hat{\lambda} \geq 0$ such that

$$
\left\langle\nabla_{z} \Lambda\left(\hat{z}, \hat{\lambda} ; \mu_{0}, \nu_{0}\right), z-\hat{z}\right\rangle \geq 0 \quad \forall z \in Z
$$

and

$$
\int_{a}^{b}\left[\mu_{0}(H(\hat{z}, \eta))-\nu_{0}([\eta, \infty))\right] \hat{\lambda}(d \eta)=0
$$

Using the nonnegativity of $\hat{\lambda}$ and the complementarity condition, we can write the chain of inequalities

$$
\begin{aligned}
& \varphi\left(\mu_{t}, \nu_{t}\right)-\varphi\left(\mu_{0}, \nu_{0}\right) \geq f\left(z_{t}\right)-\int_{a}^{b}\left[\mu_{t}\left(H\left(z_{t}, \eta\right)\right)-\nu_{t}([\eta, \infty))\right] \hat{\lambda}(d \eta)-f(\hat{z}) \\
\geq & f\left(z_{t}\right)-\int_{a}^{b}\left[\mu_{t}\left(H\left(z_{t}, \eta\right)\right)-\nu_{t}([\eta, \infty))\right] \hat{\lambda}(d \eta) \\
& -f(\hat{z})+\int_{a}^{b}\left[\mu_{0}(H(\hat{z}, \eta))-\nu_{0}([\eta, \infty))\right] \hat{\lambda}(d \eta) \\
= & \Lambda\left(z_{t}, \hat{\lambda} ; \mu_{0}, \nu_{0}\right)-\Lambda\left(\hat{z}, \hat{\lambda} ; \mu_{0}, \nu_{0}\right)-t \int_{a}^{b}\left[\gamma\left(H\left(z_{t}, \eta\right)\right)-\sigma([\eta, \infty))\right] \hat{\lambda}(d \eta) \\
= & \left\langle\nabla_{z} \Lambda\left(\hat{z}, \hat{\lambda} ; \mu_{0}, \nu_{0}\right), z_{t}-\hat{z}\right\rangle+o\left(z_{t}, \hat{z}\right)-t \int_{a}^{b}\left[\gamma\left(H\left(z_{t}, \eta\right)\right)-\sigma([\eta, \infty))\right] \hat{\lambda}(d \eta),
\end{aligned}
$$

where $o\left(z_{t}, \hat{z}\right) /\left\|z_{t}-\hat{z}\right\| \rightarrow 0$ as $t \rightarrow 0$. By the optimality condition and by the assumption that $\left\|z_{t}-\hat{z}\right\| \leq L t$, we conclude that

$$
\liminf _{t \rightarrow 0} \frac{1}{t}\left[\varphi\left(\mu_{t}, \nu_{t}\right)-\varphi\left(\mu_{0}, \nu_{0}\right)\right] \geq-\int_{a}^{b}[\gamma(H(\hat{z}, \eta))-\sigma([\eta, \infty))] \hat{\lambda}(d \eta) .
$$

Now we use the correspondence between a nonnegative measure $\hat{\lambda}$ on $[a, b]$ and a function $\hat{u} \in \mathscr{U}([a, b])$ defined as follows:

$$
\hat{u}(\eta)=\hat{\lambda}([a, \eta]), \quad \eta \in[a, b] .
$$

Changing the order of integration, as in (3.15), we obtain

$$
\begin{gathered}
\int_{a}^{b} \gamma(H(\hat{z}, \eta)) \hat{\lambda}(d \eta)=\int \hat{u}(g(\hat{z}, v)) \gamma(d v), \\
\left.\int_{a}^{b} \sigma([\eta, \infty)) \hat{\lambda}(d \eta)=\int \hat{u}(y)\right) \sigma(y) .
\end{gathered}
$$

Using the last two equations, we can rewrite (3.19) as follows:

$$
\left.\liminf _{t \rightarrow 0} \frac{1}{t}\left[\varphi\left(\mu_{t}, \nu_{t}\right)-\varphi\left(\mu_{0}, \nu_{0}\right)\right] \geq-\int \hat{u}(g(\hat{z}, v)) \gamma(d v)+\int \hat{u}(y)\right) \sigma(y) .
$$


As $\hat{\lambda}$ was an arbitrary optimal multiplier, we can take the supremum of the right-hand side over $\hat{u} \in \hat{U}$ to obtain (3.18).

We point out that the assumption of Lipschitz stability of optimal solutions, $\left\|z_{t}-\hat{z}\right\| \leq L t$, has an implicit character. In general stability studies, its fulfillment involves appropriate second order sufficient optimality conditions. In our case, due to the nature of the probability distribution functions, such an analysis is very difficult.

Finally, we obtain the directional differentiability result.

COROLLARY 3.7. Under the assumptions of Theorems 3.3 and 3.6 the optimal value function is directionally differentiable in the direction $(\gamma, \sigma)$ with the derivative

$$
\varphi^{\prime}\left(\left(\mu_{0}, \nu_{0}\right) ;(\gamma, \sigma)\right)=\sup _{\hat{u} \in \hat{U}}\left\{-\int \hat{u}(g(\hat{z}, v)) \gamma(d v)+\int \hat{u}(y) \sigma(d y)\right\} .
$$

The assumptions simplify considerably if we allow perturbations of the benchmark distribution only.

Acknowledgment. The authors are very grateful to Diethard Klatte and Alexander Shapiro for their insightful remarks and suggestions.

\section{REFERENCES}

[1] J. F. Bonnans and A. Shapiro, Perturbation Analysis of Optimization Problems, SpringerVerlag, New York, 2000.

[2] R. Cominetti, Metric regularity, tangent sets, and second-order optimality conditions, Appl. Math. Optim., 21 (1990), pp. 265-287.

[3] D. Dentcheva And A. RuszczyŃski, Optimization with stochastic dominance constraints, SIAM J. Optim., 14 (2003), pp. 548-566.

[4] D. Dentcheva And A. RuszczyńSKi, Optimality and duality theory for stochastic optimization problems with nonlinear dominance constraints, Math. Program., 99 (2004), pp. 329-350.

[5] D. Dentcheva And A. Ruszczyński, Semi-infinite probabilistic optimization: First order stochastic dominance constraints, Optimization, 53 (2004), pp. 583-601.

[6] P. C. Fishburn, Utility Theory for Decision Making, Wiley, New York, 1970.

[7] J. Hadar and W. Russell, Rules for ordering uncertain prospects, Amer. Econom. Rev., 59 (1969), pp. 25-34.

[8] R. Henrion and W. RÖmisch, Metric regularity and quantitative stability in stochastic programs with probabilistic constraints, Math. Program., 84 (1999), pp. 55-88.

[9] A. Kibzun and S. Uryasev, Differentiability of probability function, Stochastic Anal. Appl., 16 (1998), pp. 1101-1128.

[10] D. Klatte, A note on quantitative stability results in nonlinear optimization, in Proc. 19. Jahrestagung Mathematische Optimierung, K. Lommatzsch, ed., Seminarbericht 90, Humboldt-Universität, Berlin, 1987, pp. 77-86.

[11] D. Klatte and R. Henrion, Regularity and stability in nonlinear semi-infinite optimization, Nonconvex Optim. Appl., 25 (1998), pp. 69-102.

[12] D. Klatte and B. Kummer, Nonsmooth Equations in Optimization, Kluwer Academic, Dordrecht, The Netherlands, 2002.

[13] E. Lehmann, Ordered families of distributions, Ann. Math. Statist., 26 (1955), pp. 399-419.

[14] H. B. MANn AND D. R. Whitney, On a test of whether one of two random variables is stochastically larger than the other, Ann. Math. Statist., 18 (1947), pp. 50-60.

[15] K. Mosler and M. Scarsini, Eds., Stochastic Orders and Decision under Risk, Institute of Mathematical Statistics, Hayward, CA, 1991.

[16] A. PrÉKopa, Stochastic Programming, Kluwer Academic, Dordrecht, Boston, 1995.

[17] J. P. Quirk And R. SAPosnik, Admissibility and measurable utility functions, Rev. Econom. Stud., 29 (1962), pp. 140-146.

[18] S. M. Robinson, Stability theory for systems of inequalities, Part II: Differentiable nonlinear systems, SIAM J. Numer. Anal., 13 (1976), pp. 497-513.

[19] S. M. Robinson, Local epi-continuity and local optimization, Math. Program., 37 (1987), pp. 208-222.

[20] R. T. Rockafellar and R. J.-B. Wets, Variational Analysis, Springer-Verlag, Berlin, 1998. 
[21] W. Römisch, Stability of stochastic programming problems, in Stochastic Programming, A. Ruszczyński and A. Shapiro, eds., Elsevier, Amsterdam, 2003, pp. 483-554.

[22] W. Römisch And R. Schultz, Stability analysis for stochastic programs, Ann. Oper. Res., 30 (1991), pp. 241-266.

[23] W. Römisch And R. Schultz, Distribution sensitivity for certain classes of chance-constrained models with application to power dispatch, J. Optim. Theory Appl., 71 (1991), pp. 569-588.

[24] A. Ruszczyński and A. Shapiro, Eds., Stochastic Programming, Elsevier, Amsterdam, 2003.

[25] M. Shaked And J. G. Shanthikumar, Stochastic Orders and Their Applications, Academic Press, Boston, MA, 1994. 